\title{
Measurement and compensation of horizontal crabbing at the Cornell Electron Storage Ring Test Accelerator
}

\author{
M. P. Ehrlichman, * A. Chatterjee, W. Hartung, B. Heltsley, D. P. Peterson, N. Rider, \\ D. Rubin, D. Sagan, J. P. Shanks, and S. T. Wang \\ Cornell Laboratory for Accelerator-based Sciences and Education, \\ Cornell University, Ithaca, New York 14850, USA \\ (Received 7 November 2013; published 9 April 2014)
}

\begin{abstract}
In storage rings, horizontal dispersion in the rf cavities introduces horizontal-longitudinal ( $x z$ ) coupling, contributing to beam tilt in the $x z$ plane. This coupling can be characterized by a "crabbing" dispersion term $\zeta_{a}$ that appears in the normal mode decomposition of the 1-turn transfer matrix. $\zeta_{a}$ is proportional to the rf cavity voltage and the horizontal dispersion in the cavity. We report experiments at the Cornell Electron Storage Ring Test Accelerator where $x z$ coupling was explored using three lattices with distinct crabbing properties. We characterize the $x z$ coupling for each case by measuring the horizontal projection of the beam with a beam size monitor. The three lattice configurations correspond to (i) $16 \mathrm{mrad} x z$ tilt at the beam size monitor source point, (ii) compensation of the $\zeta_{a}$ introduced by one of two pairs of rf cavities with the second, and (iii) zero dispersion in $\mathrm{rf}$ cavities, eliminating $\zeta_{a}$ entirely. Additionally, intrabeam scattering is evident in our measurements of beam size vs rf voltage.
\end{abstract}

DOI: 10.1103/PhysRevSTAB.17.044002

PACS numbers: 41.75.Ht, 29.20.db, 42.72.-g, 29.27.-a

\section{INTRODUCTION}

Just as coupling of horizontal and vertical motion can result in a bunch profile that is tilted in the transverse plane, coupling of horizontal and longitudinal motion will in general produce a tilt in the horizontal-longitudinal $(x z)$ plane. The requisite $x z$ coupling can be generated by dispersion in ordinary rf accelerating cavities.

Crabbing in electron storage rings has been explored at KEKB [1]. There, specially constructed crab cavities were used to generate an $x z$ tilt of $22 \mathrm{mrad}$ at the interaction region in order to compensate for the crossing angle. Tilt angles at KEKB were measured directly using a streak camera.

Studies of $x z$ tilt have been done at the Cornell Electron Storage Ring Test Accelerator (CesrTA). At CesrTA, we measure tilt indirectly by observing the horizontal projection of the beam as rf voltage is varied. Some relevant machine parameters are shown in Table I. The CesrTA layout is shown in Fig. 1. The horizontal beam size monitor source point, the two rf cavity pairs, and the four damping wiggler triplets are highlighted. The rf straights are in close proximity to the interaction region/damping wiggler straight in the south arc. Because of the intervening hard bend magnets, there is no practical lattice solution with zero dispersion in both the rf and wiggler straights. In order to

*mpe5@cornell.edu

Published by the American Physical Society under the terms of the Creative Commons Attribution 3.0 License. Further distribution of this work must maintain attribution to the author(s) and the published article's title, journal citation, and DOI. minimize the horizontal emittance, we generally opt for zero dispersion in the wiggler straight. The result is horizontal dispersion of about $1 \mathrm{~m}$ in the rf cavities. This horizontal dispersion results in a tilt of the beam in the $x z$ plane by an amount that depends on the total $\mathrm{rf}$ accelerating voltage.

The horizontal beam size monitor at CesrTA measures the projection of the beam into the horizontal lab frame coordinate. A tilt of the beam in the $x z$ plane manifests itself as an increase in the measured horizontal beam size. Bunch lengths in CesrTA are typically $10 \mathrm{~mm}$ and bunch widths are typically $150 \mu \mathrm{m}$. Even small amounts of tilt can result in a significantly larger measured horizontal size.

The largest inferred tilt at the beam size monitor source point is $16 \mathrm{mrad}$. The tilt is modulated by the horizontal phase advance, and the largest tilt in our lattice model is 47 mrad.

TABLE I. CesrTA machine parameters for crabbing studies. $\nu_{x, y, s}=2 \pi Q_{x, y, s}$ is used in this paper.

\begin{tabular}{lcc}
\hline \hline Beam energy $(\mathrm{GeV})$ & $E_{0}$ & 2.085 \\
\hline Circumference $(\mathrm{m})$ & $L$ & 768 \\
RF frequency $(\mathrm{MHz})$ & $\omega_{\mathrm{rf}}$ & $2 \pi \times 500$ \\
Transverse damping time $(\mathrm{ms})$ & $\tau$ & 56.6 \\
Momentum compaction & $\alpha_{p}$ & 0.0068 \\
Nominal RF voltage $(\mathrm{MV})$ & $V_{\mathrm{rf}}$ & 6.3 \\
Synchrotron tune & $Q_{s}$ & 0.065 \\
Horizontal tune & $Q_{x}$ & 14.624 \\
Vertical tune & $Q_{y}$ & 9.590 \\
Horizontal emittance $(\mathrm{nm} \cdot \mathrm{rad})$ & $\epsilon_{a}$ & $\sim 3$ \\
Vertical emittance $(\mathrm{pm} \cdot \mathrm{rad})$ & $\epsilon_{b}$ & $\sim 5$ to $\sim 15$ \\
\hline \hline
\end{tabular}




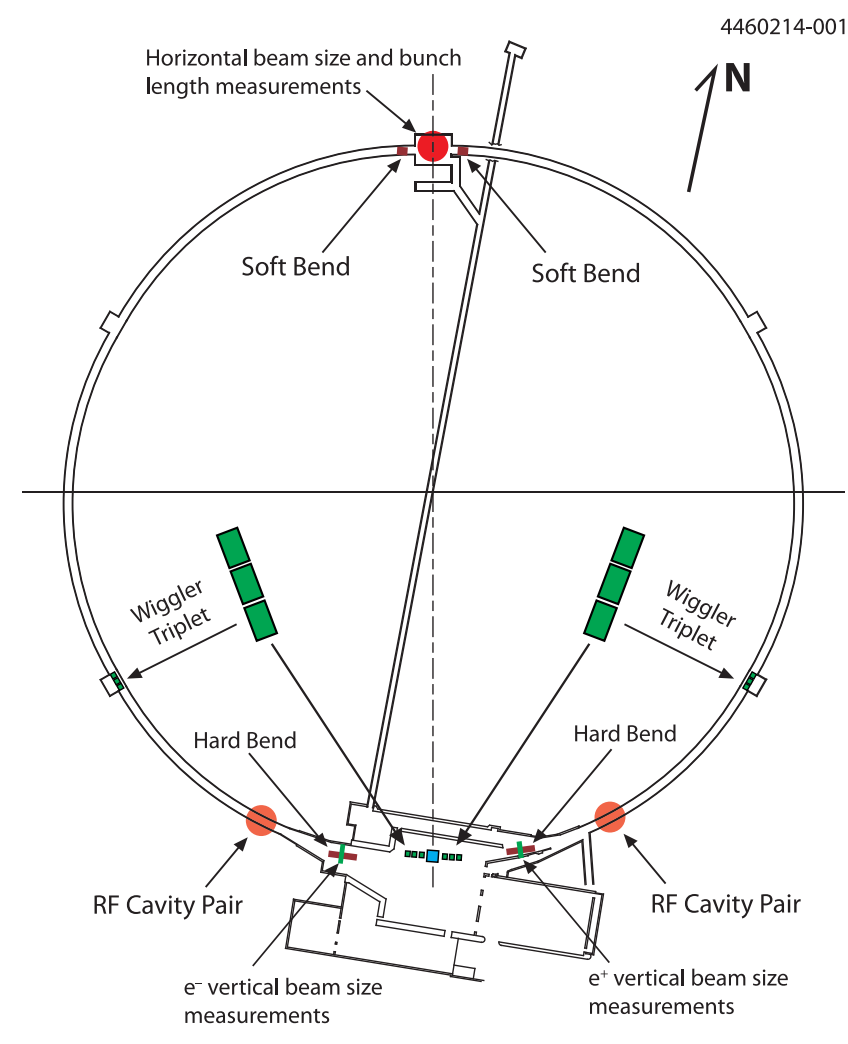

FIG. 1. The configuration of the Cornell Electron Storage Ring Test Accelerator [2].

In this paper, we describe recent experiments at CesrTA to measure and correct the $x z$ tilt, and the theoretical basis for our correction techniques. The calculations presented here were conducted using the BMAD accelerator simulation suite [3].

Adjusting the rf voltage also changes the bunch length and hence the particle density, which in turn changes the amount of IBS blow up. In addition to measuring the effect of beam tilt, we observe intrabeam scattering (IBS) effects, as we measure vertical as well as horizontal size.

In Sec. II we show with a simple model how dispersion in the rf cavities leads to a beam that is tilted in the $x z$ plane.

In Sec. III, we develop a parametrization of the one-turn matrix and a numerical method for obtaining it. From this parametrization, we extract the tilt and projected size of beams in arbitrary coupling conditions. This section's intent is to give the reader a more rigorous and complete picture of the parametrization and to explain how it describes the beam envelope.

In Sec. IV, we present two methods for eliminating the tilt: (i) canceling the crabbing dispersion $\zeta_{a}$ by adjusting the horizontal phase advance between the two pairs of $\mathrm{rf}$ cavities; (ii) constraining the optics so that the dispersion is zero in the rf straights. This necessarily results in nonzero dispersion in the wiggler straight, hence for operation in these optics, 6 of the 12 damping wigglers must be turned off.
In Sec. V, we test our formalism for calculating beam sizes, as well as our tilt-mitigating lattices, by measuring the beam size as a function of rf voltage.

Manipulation of $x z$ coupling can be useful for both collider damping rings and light sources. In a collider, $x z$ coupling can be used to achieve full head-on collisions. The technique is being considered as part of a future LHC luminosity upgrade [4]. In light sources, $x z$ coupled beams have been proposed as a method for obtaining subpicosecond $\mathrm{x}$-ray pulses from a storage ring [5].

Typically, $x z$ coupling is obtained using crab cavities. Crab cavities use the magnetic field of a $\mathrm{TM}_{110}$ horizontal dipole mode to apply a phase-dependent transverse kick to the beam. The formalism presented here is a potentially simpler way to manipulate $x z$ coupling in an accelerator. It does not require new technology and is based on elementary beam optics.

\section{BRIEF DESCRIPTION OF CRABBING DUE TO DISPERSION IN A RF CAVITY}

$\mathrm{Rf}$ cavities are present in a storage ring to restore energy to the beam that is lost due to synchrotron radiation. In machines operating above transition, the rf phase is set so that the lower-energy particles which arrive at the rf cavity sooner receive a bigger energy kick, while the higher-energy particles which arrive later receive a smaller kick. The kick received is

$$
\frac{\Delta E}{E_{0}}=\frac{e V_{\mathrm{rf}}}{E_{0}} \sin \left(\Psi_{0}-\Delta \Psi\right),
$$

where $E_{0}$ is the beam energy, $e$ is the electric charge, $V_{\mathrm{rf}}$ is the peak cavity voltage, $\Psi_{0}$ is the nominal synchrotron phase, and

$$
\Delta \Psi=\frac{z \omega_{\mathrm{rf}}}{\beta_{r} c},
$$

where $z$ is the longitudinal coordinate relative to the reference particle, $\omega_{\mathrm{rf}}$ is the $\mathrm{rf}$ frequency, $c$ is the speed of light, and $\beta_{r}$ is the relativistic beta. Particles at the head of the bunch have a positive $z$. Above transition, $\frac{\pi}{2}<\Psi_{0}<\frac{3 \pi}{2}$, and typically $\Psi_{0} \sim \pi$. Expanding Eq. (1) about $\Psi_{0}=\pi$ yields,

$$
\frac{\Delta E}{E_{0}} \approx \tilde{V} z
$$

where

$$
\tilde{V} \equiv \frac{e V_{r f} \omega_{\mathrm{rf}}}{\beta_{r} c E_{0}} .
$$

The position $x$ of a particle has contributions from betatron motion and dispersion $\eta_{x}$, 


$$
x=x_{\beta}+\delta \eta_{x}
$$

where $\delta$ is the relative energy deviation of the particle. In a "zero-length" cavity, a particle receives an energy kick $\Delta E$, but its instantaneous position does not change. So,

$$
\Delta x=0=\Delta x_{\beta}+\frac{\Delta E}{E_{0}} \eta_{x} \rightarrow \Delta x_{\beta}=-\frac{\Delta E}{E_{0}} \eta_{x} .
$$

Suppose that $\eta_{x} \neq 0$ in the cavity. The formula for the change in the closed orbit at location $s$ due to a displacement $\Delta x_{0}$ at location $s_{0}$ is

$$
x(s)=-\Delta x_{0} \sqrt{\beta_{x}(s) \beta_{x 0}} \frac{\gamma_{x 0} \sin \left(\Delta \phi(s)-\pi Q_{x}+\phi_{\alpha 0}\right)}{2 \sin \pi Q_{x}},
$$

where $s$ is the location of the reference particle in the accelerator, $\Delta \phi(s)$ is the phase advance from $s_{0}$ to $s, \beta_{x}(s)$ is the horizontal Twiss parameter at $s, \phi_{\alpha 0}=\arcsin \frac{-\alpha_{x 0}}{\sqrt{1+\alpha_{x 0}^{2}}}$, and $\beta_{x 0}, \alpha_{x 0}$, and $\gamma_{x 0}$ are the horizontal Twiss parameters at $s=s_{0}$.

Setting $\Delta x_{\beta}=\Delta x_{0}$ and $\eta_{x}=\eta_{x 0}$ in Eq. (6), and combining Eqs. (3), (6), and (7), and dividing by $z$ yields the $x z$-tilt angle,

$$
\begin{aligned}
\theta_{x z}(s) & =\tan \frac{x(s)}{z} \\
& \approx \tilde{V} \eta_{x 0} \sqrt{\beta_{x}(s) \beta_{x 0}} \frac{\gamma_{x 0} \sin \left(\Delta \phi(s)-\pi Q_{x}+\phi_{\alpha 0}\right)}{2 \sin \pi Q_{x}}
\end{aligned}
$$

Equation (8) gives the $x z$ tilt at some location $s$ due to dispersion in a rf cavity at $s_{0}$. A similar treatment would reveal the tilt due to having finite $\eta_{x}^{\prime}$ in a rf cavity.

Inspecting Eq. (8) we see that if one were to follow the beam around the ring, the tilt angle would be observed to oscillate as the betatron phase advances, and the amount of tilt is proportional to $\sqrt{\beta_{x}}$ at the observation point.

Later, in Sec. III D, equations are derived for the beam tilt in terms of the parametrization of the one-turn matrix. Equation (8) agrees with these later results in the appropriate limits.

\section{THEORY}

Consider the one-turn map $\mathbf{T}_{4}$ for four-dimensional phase space $\left(x, p_{x}, y, p_{y}\right)$. The Jacobian of the map is a symplectic $4 \times 4$ matrix. The Edwards-Teng parametrization expresses $\mathbf{T}_{4}$ in terms of 10 parameters [6]: two normal-mode phase advances, four normal-mode Twiss parameters, and four coupling parameters. The coupling parameters describe how the normal-mode coordinates $\left(a, p_{a}, b, p_{b}\right)$ transform into lab-frame coordinates $\left(x, p_{x}, y, p_{y}\right)$. Three of the four coupling parameters can be measured directly in CESR, which allows for optics correction [7]. Additionally, in the limit of linear optics and Gaussian beams, normal-mode emittances are well-defined invariants.

The Edwards-Teng parametrization is incomplete in that it ignores longitudinal motion. In [8], Ohmi, Hirata, and Oide extend the Edwards-Teng parametrization to the full $6 \times 6$ transfer matrix T. T is the one-turn map for sixdimensional phase space $\left(x, p_{x}, y, p_{y}, z, p_{z}\right)$. It is described by 21 parameters: three normal-mode phase advances, six normal-mode Twiss parameters, and twelve coupling parameters.

The $6 \times 6$ parametrization of the one-turn map is useful when there is significant coupling between the longitudinal motion and the transverse motion. The orientation of the beam envelope can be written in terms of the coupling parameters. With this description, the coupling properties of the ring can be adjusted or corrected by varying these parameters. Additionally, the normal-mode Twiss parameters $\beta_{a}, \alpha_{a}, \beta_{b}, \alpha_{b}, \beta_{c}$, and $\alpha_{c}$, and normal-mode emittances $\epsilon_{a}, \epsilon_{b}$, and $\epsilon_{c}$ are well-defined quantities.

In this section we present the $6 \times 6$ parametrization in a format that is convenient for investigating tilt in the $x z$ plane, and we also describe a numerical method for obtaining it. Our description differs from that in [8] in that we only use real-valued quantities. We extend the formalism by defining "normalized" coupling parameters which simplify the expressions for the beam tilt and beam size.

\section{A. The $6 \times 6$ parametrization}

The Edwards-Teng parametrization is extended to the $6 \times 6$ case via the "dispersion matrix" $\mathbf{H}$ introduced in [8],

$$
\mathbf{T}=\mathbf{H V U V} \mathbf{V}^{\dagger} \mathbf{H}^{\dagger},
$$

where

$$
\begin{gathered}
\mathbf{U}=\left(\begin{array}{ccc}
\mathbf{U}_{a} & \mathbf{0} & \mathbf{0} \\
\mathbf{0} & \mathbf{U}_{b} & \mathbf{0} \\
\mathbf{0} & \mathbf{0} & \mathbf{U}_{c}
\end{array}\right), \\
\mathbf{V}=\left(\begin{array}{ccc}
\mu \mathbf{I}_{2} & \mathbf{V}_{2} & 0 \\
-\mathbf{V}_{2}^{\dagger} & \mu \mathbf{I}_{2} & 0 \\
0 & 0 & \mathbf{I}_{2}
\end{array}\right), \\
\mathbf{H}=\left(\begin{array}{ccc}
\left(1-\frac{\left|\mathbf{H}_{a}\right|}{1+\rho}\right) \mathbf{I}_{2} & -\frac{\mathbf{H}_{a} \mathbf{H}_{b}^{\dagger}}{1+\rho} & \mathbf{H}_{a} \\
-\frac{\mathbf{H}_{b} \mathbf{H}_{a}^{\dagger}}{1+\rho} & \left(1-\frac{\left|\mathbf{H}_{b}\right|}{1+\rho}\right) \mathbf{I}_{2} & \mathbf{H}_{b} \\
-\mathbf{H}_{a}^{\dagger} & -\mathbf{H}_{b}^{\dagger} & \rho \mathbf{I}_{2}
\end{array}\right) .
\end{gathered}
$$


The $2 \times 2$ submatrices are defined as

$$
\mathbf{V}_{2}=\left(\begin{array}{ll}
v_{11} & v_{12} \\
v_{21} & v_{22}
\end{array}\right), \quad \mathbf{H}_{a, b}=\left(\begin{array}{cc}
\zeta_{a, b} & \eta_{a, b} \\
\zeta_{a, b}^{\prime} & \eta_{a, b}^{\prime}
\end{array}\right),
$$

and $\mathbf{I}_{2}$ is the $2 \times 2$ identity matrix.

$\mathbf{U}$ describes the motion of the particles in normal-mode coordinates. $\mathbf{V}$ and $\mathbf{H}$ describe how the normal-modes couple into lab coordinates.

$\eta_{a, b}$ and $\eta_{a, b}^{\prime}$ are referred to as normal-mode dispersions and their derivatives. $\zeta_{a, b}$ and $\zeta_{a, b}^{\prime}$ are referred to as normalmode crabbing dispersions and their derivatives. For the $a$-mode,

$$
\mathbf{U}_{a}=\left(\begin{array}{cc}
\cos \theta_{a}+\alpha_{a} \sin \theta_{a} & \beta_{a} \sin \theta_{a} \\
-\gamma_{a} \sin \theta_{a} & \cos \theta_{a}-\alpha_{a} \sin \theta_{a}
\end{array}\right),
$$

where $\theta_{a}$ is the phase advance per turn of the $a$-mode. There are similar equations for $\mathbf{U}_{b}$ and $\mathbf{U}_{c}$.

For some $2 n \times 2 n$ matrix $\mathbf{M}_{2 n}$, the symplectic conjugate $\mathbf{M}_{2 n}^{\dagger}$ is defined as

$$
\mathbf{M}_{2 n}^{\dagger}=-\mathbf{S}_{2 n} \mathbf{M}_{2 n}^{T} \mathbf{S}_{2 n},
$$

where $\mathbf{S}_{2 n}$ is a $2 n \times 2 n$ matrix whose $2 \times 2$ diagonal blocks are

$$
\mathbf{S}_{2}=\left(\begin{array}{cc}
0 & 1 \\
-1 & 0
\end{array}\right)
$$

Note that if $\mathbf{M}_{2 n}$ is symplectic, then $\mathbf{M}_{2 n}^{-1}=\mathbf{M}_{2 n}^{\dagger}$. Superscript ${ }^{T}$ denotes the matrix transpose.

\section{B. Computing the $6 \times 6$ parametrization}

In the general case, the normal-mode decomposition can be obtained via the eigen decomposition. In [9], the full turn matrix $\mathbf{T}$ is decomposed into

$$
\mathbf{A}=\mathbf{H V B P}=\left(\begin{array}{ccc}
\left(\mu\left(1-\frac{\left|\mathbf{H}_{a}\right|}{1+\rho}\right) \mathbf{I}_{2}+\frac{\mathbf{H}_{a} \mathbf{H}_{b}^{\dagger} \mathbf{V}_{2}^{\dagger}}{1+\rho}\right) \mathbf{B}_{a} \mathbf{P}_{a} & \left(\left(1-\frac{\left|\mathbf{H}_{a}\right|}{1+\rho}\right) \mathbf{V}_{2}-\mu \frac{\mathbf{H}_{a} \mathbf{H}_{b}^{\dagger}}{1+\rho}\right) \mathbf{B}_{b} \mathbf{P}_{b} & \mathbf{H}_{a} \mathbf{B}_{c} \mathbf{P}_{c} \\
-\left(\left(1-\frac{\left|\mathbf{H}_{b}\right|}{1+\rho}\right) \mathbf{V}_{2}^{\dagger}+\mu \frac{\mathbf{H}_{b} \mathbf{H}_{a}^{\dagger}}{1+\rho}\right) \mathbf{B}_{a} \mathbf{P}_{a} & \left(\mu\left(1-\frac{\left|\mathbf{H}_{b}\right|}{1+\rho}\right) \mathbf{I}_{2}-\frac{\mathbf{H}_{b} \mathbf{H}_{a}^{\dagger} \mathbf{V}_{2}}{1+\rho}\right) \mathbf{B}_{b} \mathbf{P}_{b} & \mathbf{H}_{b} \mathbf{B}_{c} \mathbf{P}_{c} \\
\left(-\mu \mathbf{H}_{a}^{\dagger}+\mathbf{H}_{b}^{\dagger} \mathbf{V}_{2}^{\dagger}\right) \mathbf{B}_{a} \mathbf{B}_{a} & \left(-\mu \mathbf{H}_{b}^{\dagger}-\mathbf{H}_{a}^{\dagger} \mathbf{V}_{2}\right) \mathbf{B}_{b} \mathbf{B}_{b} & \rho \mathbf{B}_{c} \mathbf{P}_{c}
\end{array}\right) .
$$

Because $\left|\mathbf{B}_{c} \mathbf{P}_{c}\right|=1$, it is clear from Eq. (25) that $\rho$ is the square root of the determinant of the lower right $2 \times 2$ block of $\mathbf{A}$. It is then simple to obtain $\mathbf{B}_{c} \mathbf{P}_{c}$, as well as $\mathbf{H}_{a}$ and $\mathbf{H}_{b}$, which completely defines $\mathbf{H}$. This allows VBP to be obtained via $\mathbf{H}^{\dagger} \mathbf{A}$. Similar steps then reveal $\mu$ and $\mathbf{V}_{2}$, defining $\mathbf{V}$ and allowing $\mathbf{B P}$ to be obtained. $\psi_{a}, \psi_{b}$, and $\psi_{c}$ are selected to make the $(1,2),(3,4)$, and $(5,6)$ elements of $\mathbf{V}^{\dagger} \mathbf{H}^{\dagger} \mathbf{A} \mathbf{P}^{\dagger}$ zero. where $\mathbf{A}$ and $\mathbf{R}$ are real and symplectic. ${ }^{1} \mathbf{R}$ is block diagonal,

$$
\mathbf{R}=\operatorname{diag}\left(\mathbf{R}_{a}, \mathbf{R}_{b}, \mathbf{R}_{c}\right)
$$

where

$$
\mathbf{R}_{a, b, c}=\left(\begin{array}{cc}
\cos \theta_{a, b, c} & \sin \theta_{a, b, c} \\
-\sin \theta_{a, b, c} & \cos \theta_{a, b, c}
\end{array}\right) .
$$

A can be further decomposed to separate Twiss and coupling information [8],

$$
\mathbf{A}=\text { HVBP. }
$$

$\mathbf{H}$ and $\mathbf{V}$ are the "dispersion matrix" and "Teng matrix" as defined above and PB contains the Twiss information,

$$
\begin{gathered}
\mathbf{P}=\operatorname{diag}\left(\mathbf{P}_{a}, \mathbf{P}_{b}, \mathbf{P}_{c}\right), \\
\mathbf{P}_{a, b, c}=\left(\begin{array}{cc}
\cos \psi_{a, b, c} & \sin \psi_{a, b, c} \\
-\sin \psi_{a, b, c} & \cos \psi_{a, b, c}
\end{array}\right), \\
\mathbf{B}=\operatorname{diag}\left(\mathbf{B}_{a}, \mathbf{B}_{b}, \mathbf{B}_{c}\right), \\
\mathbf{B}_{a, b, c}=\left(\begin{array}{cc}
\sqrt{\beta_{a, b, c}} & 0 \\
-\frac{\alpha_{a, b, c}}{\sqrt{\beta_{a, b, c}}} & \frac{1}{\sqrt{\beta_{a, b, c}}}
\end{array}\right) .
\end{gathered}
$$

$\mathbf{P}$ is not an observable. The three phases $\psi_{a}, \psi_{b}$, and $\psi_{c}$ are arbitrary and chosen to give $\mathbf{B}$ the desired form.

Equations (11), (12), (21), and (23) are combined to write

Finally, the full turn matrix is written as

$$
\mathbf{T}=\mathbf{H V B P R} \mathbf{P}^{\dagger} \mathbf{B}^{\dagger} \mathbf{V}^{\dagger} \mathbf{H}^{\dagger}
$$

This eigen decomposition becomes a normal-mode decomposition [7] by writing

$$
\mathbf{U}=\mathbf{B P R} \mathbf{P}^{\dagger} \mathbf{B}^{\dagger}
$$

so that

\footnotetext{
${ }^{1} \mathbf{A}$ here is $\mathbf{N}$ from Eq. 44 in Ref. [9].
} 


\section{$\mathbf{T}=\mathbf{H V U V} \mathbf{V}^{\dagger} \mathbf{H}^{\dagger}$}

\section{Projecting normal-mode coordinates into lab frame coordinates}

Lab frame coordinates $\mathbf{x}=\left(x, p_{x}, y, p_{y}, z, p_{z}\right)$ and normal-mode coordinates $\mathbf{a}=\left(a, p_{a}, b, p_{b}, c, p_{c}\right)$ are connected by

$$
\mathbf{x}=\mathbf{H V a} .
$$

Writing out $\mathbf{H V}$,

$$
\mathbf{H V}=\left(\begin{array}{ccc}
\mu\left(1-\frac{\left|\mathbf{H}_{a}\right|}{1+a}\right) \mathbf{I}_{2}+\frac{\mathbf{H}_{a} \mathbf{H}_{b}^{\dagger} \mathbf{V}_{2}^{\dagger}}{1+a} & \left(1-\frac{\left|\mathbf{H}_{a}\right|}{1+a}\right) \mathbf{V}_{2}-\mu \frac{\mathbf{H}_{a} \mathbf{H}_{b}^{\dagger}}{1+a} & \mathbf{H}_{a} \\
-\left(1-\frac{\left|\mathbf{H}_{b}\right|}{1+a}\right) \mathbf{V}_{2}^{\dagger}+\mu \frac{\mathbf{H}_{b} \mathbf{H}_{a}^{\dagger}}{1+a} & \mu\left(1-\frac{\left|\mathbf{H}_{b}\right|}{1+a}\right) \mathbf{I}_{2}-\frac{\mathbf{H}_{b} \mathbf{H}_{a}^{\dagger} \mathbf{V}_{2}}{1+a} & \mathbf{H}_{b} \\
-\mu \mathbf{H}_{a}^{\dagger}+\mathbf{H}_{b}^{\dagger} \mathbf{V}_{2}^{\dagger} & -\mu \mathbf{H}_{b}^{\dagger}-\mathbf{H}_{a}^{\dagger} \mathbf{V}_{2} & a \mathbf{I}_{2}
\end{array}\right),
$$

we see that the $c$ normal mode is coupled into the $x$ lab frame coordinate via $\mathbf{H}_{a}$.

As shown in [9], the eigenvectors of the full turn matrix $\mathbf{M}$ are the same as the eigenvectors of $\Sigma \mathbf{S}$, where $\Sigma$ is the matrix of second order moments of a Gaussian distribution matched to the machine lattice functions. The $\Sigma$ matrix can be obtained from

$$
\Sigma \mathbf{S}=\mathbf{A D A}^{\dagger}
$$

where,

$$
\mathbf{D}=\left(\begin{array}{cccccc}
0 & \epsilon_{a} & 0 & 0 & 0 & 0 \\
-\epsilon_{a} & 0 & 0 & 0 & 0 & 0 \\
0 & 0 & 0 & \epsilon_{b} & 0 & 0 \\
0 & 0 & -\epsilon_{b} & 0 & 0 & 0 \\
0 & 0 & 0 & 0 & 0 & \epsilon_{c} \\
0 & 0 & 0 & 0 & -\epsilon_{c} & 0
\end{array}\right),
$$

and $\epsilon_{a}, \epsilon_{b}$, and $\epsilon_{c}$ are the three normal-mode emittances. Note that normal-mode emittances and eigenmode emittances are equivalent.

Normalizing the $\mathbf{H}$ and $\mathbf{V}$ coupling matrices by the $\beta$-functions allows us to write simplified expressions for the crabbing angles and beam sizes [7]. We define,

$$
\begin{aligned}
& \overline{\mathbf{H}}=\mathbf{B}^{\dagger} \mathbf{H B} \\
& \overline{\mathbf{V}}=\mathbf{B}^{\dagger} \mathbf{V B} .
\end{aligned}
$$

The exact angle of the major axis of an ellipse in the $i j$ plane, where $i$ and $j$ could be any of $x, p_{x}, y, p_{y}, z$, or $p_{z}$ is

$$
\theta_{i j}=\frac{1}{2} \arctan \frac{2 \sigma_{i j}}{\sigma_{i i}-\sigma_{j j}},
$$

where $\sigma_{i j}, \sigma_{i i}$, and $\sigma_{j j}$ are elements of the beam $\Sigma$ matrix.

The tilt of the beam in some plane in terms of the HVBP parameters can be found by combining Eqs. (20), (31), (33), (34), and (35).

To first order in $\overline{\mathbf{H}}_{a}, \overline{\mathbf{H}}_{b}$, and $\overline{\mathbf{V}}_{2}$ the horizontal crabbing angle $\theta_{x z}$ and vertical crabbing angle $\theta_{y z}$ are

$$
\begin{aligned}
\theta_{x z} & \approx \sqrt{\beta_{a} \beta_{c}} \frac{\epsilon_{c} \overline{\mathbf{H}}_{15}-\epsilon_{a} \overline{\mathbf{H}}_{26}}{\epsilon_{c} \beta_{c}-\epsilon_{a} \beta_{a}}, \\
\theta_{y z} & \approx \sqrt{\beta_{b} \beta_{c}} \frac{\epsilon_{c} \overline{\mathbf{H}}_{35}-\epsilon_{b} \overline{\mathbf{H}}_{46}}{\epsilon_{c} \beta_{c}-\epsilon_{b} \beta_{b}} .
\end{aligned}
$$

In the limits $\epsilon_{c} \gg \epsilon_{a}$ and $\epsilon_{c} \gg \epsilon_{b}$,

$$
\begin{aligned}
& \theta_{x z} \approx \sqrt{\frac{\beta_{a}}{\beta_{c}}} \overline{\mathbf{H}}_{15}=\zeta_{a}-\frac{\alpha_{c}}{\beta_{c}} \eta_{a}, \\
& \theta_{y z} \approx \sqrt{\frac{\beta_{b}}{\beta_{c}}} \overline{\mathbf{H}}_{35}=\zeta_{b}-\frac{\alpha_{c}}{\beta_{c}} \eta_{b} .
\end{aligned}
$$

In CesrTA, $\epsilon_{c} \sim 10^{-6} \mathrm{~m}, \epsilon_{a} \sim 10^{-9} \mathrm{~m}$, and $\epsilon_{b} \sim 10^{-12} \mathrm{~m}$, so the approximation is valid.

The amplitude of $\alpha_{c}$ in a storage ring can be approximated by $\left|\tan \left(\pi Q_{s}\right)\right|$, where $Q_{s}$ is the synchrotron tune. Because $Q_{s}$ is typically near zero, $\beta_{c}$ is relatively constant and can be estimated by $\beta_{c} \approx \frac{\alpha_{p} L}{\sin \nu_{s}}$. Using values from Table I, the amplitude of $\alpha_{c}$ is 0.21 and $\beta_{c}$ is $13.1 \mathrm{~m}$.

Equation (38) shows that, to leading order, there are two contributions to the $x z$ tilt angle. It will be shown in Sec. III D that the first contribution can be generated by having nonzero dispersion in the rf cavities. The other 
contribution is present in all storage rings and is proportional to the local dispersion and longitudinal Twiss $\alpha_{c}$.

The projection of the beam envelope into the lab frame is obtained from the $(1,1),(3,3)$, and $(5,5)$ elements of $\Sigma$. These expressions are, in general, quite complicated. To second order in $\overline{\mathbf{H}}_{a}, \overline{\mathbf{H}}_{b}$, and $\overline{\mathbf{V}}_{2}$ the beam sizes are

$$
\begin{aligned}
\sigma_{x}^{2} \approx & \beta_{a}\left[\left(1-2 \frac{\left|\overline{\mathbf{H}}_{a}\right|}{1+\rho}\right) \mu^{2} \epsilon_{a}\right. \\
& \left.+\left(1-2 \frac{\left|\overline{\mathbf{H}}_{a}\right|}{1+\rho}\right)\left(\bar{v}_{11}^{2}+\bar{v}_{12}^{2}\right) \epsilon_{b}+\left(\bar{\zeta}_{a}^{2}+\bar{\eta}_{a}^{2}\right) \epsilon_{c}\right], \\
\sigma_{y}^{2} \approx & \beta_{b}\left[\left(1-2 \frac{\left|\overline{\mathbf{H}}_{b}\right|}{1+\rho}\right) \mu^{2} \epsilon_{b}\right. \\
& \left.+\left(1-2 \frac{\left|\overline{\mathbf{H}}_{b}\right|}{1+\rho}\right)\left(\bar{v}_{12}^{2}+\bar{v}_{22}^{2}\right) \epsilon_{a}+\left(\bar{\zeta}_{b}^{2}+\bar{\eta}_{b}^{2}\right) \epsilon_{c}\right], \\
\sigma_{z}^{2} \approx & \beta_{c}\left[a^{2} \epsilon_{c}+\left(\bar{\eta}_{a}^{2}+\bar{\eta}_{a}^{\prime 2}\right) \mu^{2} \epsilon_{a}+\left(\bar{\eta}_{b}^{2}+\bar{\eta}_{b}^{\prime 2}\right) \mu^{2} \epsilon_{b}\right] .
\end{aligned}
$$

Figure 2 shows the $x z$ projection at the source point for the horizontal beam size monitor. The width of the $x$ projection of the beam is $227 \mu \mathrm{m}$. Figure 2 also indicates the major axis of the beam envelope and the tilt angle $\theta_{x z}$.

Figure 3 shows $\theta_{x z}$ evaluated for a nominal CesrTA lattice using Eq. (38).

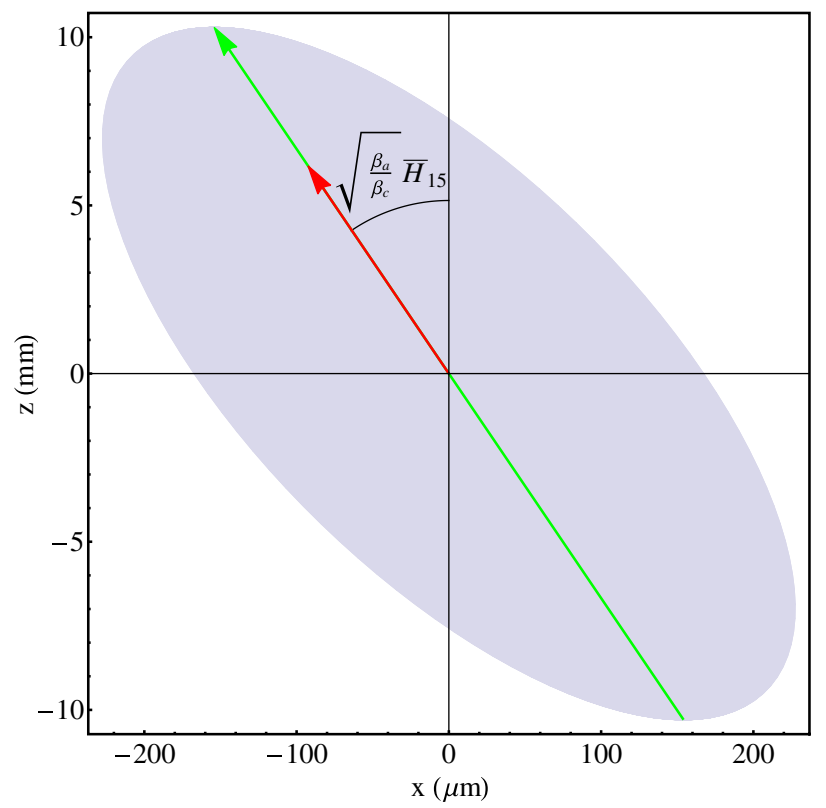

FIG. 2. Projection of the beam envelope into the $x z$ plane with a crabbing angle of -15 mrad. The green arrow is the major axis of the ellipse. The red arrow is crabbing angle obtained from the HVBP parametrization. Note that the horizontal and vertical axes have very different scales in order to make the beam tilt discernible; a side effect is to make it appear as if the green arrow is not along the major axis.

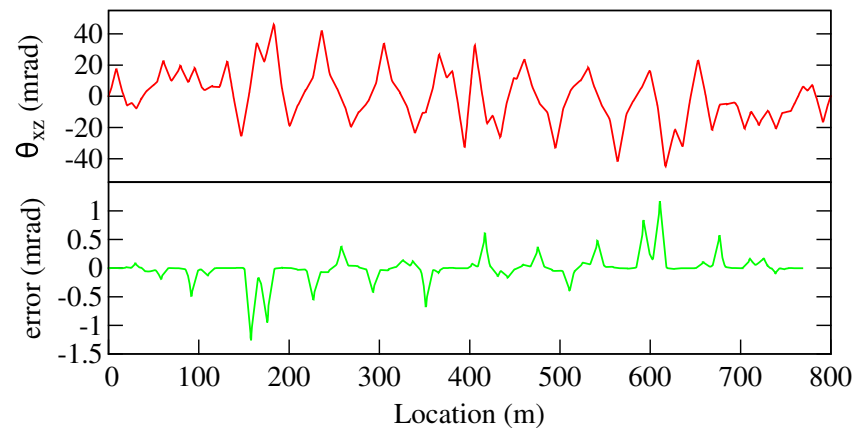

FIG. 3. Top: $\theta_{x z}$ crabbing angle calculated using Eq. (38). Bottom: difference between $\theta_{x z}$ obtained from Eq. (38) and the actual tilt of the beam envelope.

\section{D. $\mathbf{H}_{a}$ as a function of cavity voltage in a simplified model}

In the case where $x y$ and $y z$ coupling can be ignored, the normal-mode decomposition can be written in terms of the $2 \times 2$ blocks of the 1-turn matrix. In this case, $\mathbf{V}_{2}=\mathbf{0}$, $\mathbf{H}_{b}=\mathbf{0}, \mu=1$. It is illuminating to consider this case because it demonstrates how the presence of dispersion in the rf cavities generates beam tilt, and it also suggests a method for mitigating the tilt in rings where there is tunable betatron phase advance between two or more sets of $\mathrm{rf}$ cavities.

The full turn matrix has the form

$$
\mathbf{T}=\left(\begin{array}{ccc}
\mathbf{M} & \mathbf{0} & \mathbf{m} \\
\mathbf{0} & \mathbf{Y} & \mathbf{0} \\
\mathbf{n} & \mathbf{0} & \mathbf{N}
\end{array}\right),
$$

where $\mathbf{M}, \mathbf{m}, \mathbf{Y}, \mathbf{n}$, and $\mathbf{N}$ are $2 \times 2$ matrices and $\mathbf{0}$ is the $2 \times 2$ matrix of zeros.

The solution for $\mathbf{H}$ is given by,

$$
\begin{gathered}
a=\sqrt{\frac{1}{2}+\frac{1}{2} \sqrt{\frac{\operatorname{Tr}(\mathbf{M}-\mathbf{N})^{2}}{\operatorname{Tr}(\mathbf{M}-\mathbf{N})^{2}+4\left|\mathbf{m}+\mathbf{n}^{\dagger}\right|}},} \\
\mathbf{H}_{a}=\frac{\mathbf{m}+\mathbf{n}^{\dagger}}{a \sqrt{\operatorname{Tr}(\mathbf{M}-\mathbf{N})^{2}+4\left|\mathbf{m}+\mathbf{n}^{\dagger}\right|}},
\end{gathered}
$$

where $\operatorname{Tr}(\cdot)$ is the matrix trace.

The presence of horizontal dispersion in CesrTA's rf cavities introduces $x z$ and $p_{x} z$ coupling throughout the ring. This coupling causes $\zeta_{a}$ and $\zeta_{a}^{\prime}$ to be nonzero.

To see how these coupling terms arise, consider a simplified model of a flat storage ring that is perfectly aligned so there is no $x y$ nor $y z$ coupling. The storage ring has a rf cavity located at Point 1 , and we wish to determine $\zeta_{a}$ at Point 0 . The full turn matrix is given by

$$
\mathbf{T}=\mathbf{T}_{10} \mathbf{T}_{\mathrm{RF}} \mathbf{T}_{01},
$$


where $\mathbf{T}_{01}$ is the map from Point 0 to Point 1 , and $\mathbf{T}_{\mathrm{RF}}$ is the map for the rf cavity. For simplicity, assume $\beta_{x}$ is uniform around the ring and $\alpha_{x}=0$. To obtain $\mathbf{T}_{01}$, populate Eq. (43) with

$$
\mathbf{M}_{01}=\left(\begin{array}{cc}
\cos \Delta \phi_{01} & \beta_{x} \sin \Delta \phi_{01} \\
-\frac{1}{\beta_{x}} \sin \Delta \phi_{01} & \cos \Delta \phi_{01}
\end{array}\right)
$$

where $\Delta \phi_{01}=\phi_{1}-\phi_{0}$ is the horizontal phase advance from 0 to 1 , and

$$
\mathbf{m}_{01}=\left(\begin{array}{cc}
0 & \eta_{x 1} \\
0 & \eta_{x 1}^{\prime}
\end{array}\right)-\mathbf{M}_{01}\left(\begin{array}{cc}
0 & \eta_{x 0} \\
0 & \eta_{x 0}^{\prime}
\end{array}\right)
$$

where $\eta_{x}$ and $\eta_{x}^{\prime}$ are the ordinary dispersion and its derivative, i.e., the dispersion in the limit of zero rf voltage. Also define,

$$
\mathbf{N}_{01}=\left(\begin{array}{cc}
1 & L_{01} \alpha_{01}^{p} \\
0 & 1
\end{array}\right)
$$

where $L_{01}$ and $\alpha_{01}^{p}$ are the fraction of the total circumference from 0 to 1 and effective momentum compaction between 0 and 1, respectively. From the symplecticity of $\mathbf{M}$ we have,

$$
\mathbf{n}_{01}^{T}=\mathbf{M}_{01}^{T} \mathbf{S}_{2} \mathbf{m}_{01} \mathbf{N}_{01}^{-1} \mathbf{S}_{2}
$$

Because the ring is flat and ideal, $\mathbf{Y}_{01}=\mathbf{I}_{2}$.

The transfer matrix $\mathbf{T}_{\mathrm{rf}}$ for the rf cavity is given by

$$
\begin{gathered}
\mathbf{M}_{\mathrm{rf}}=\mathbf{Y}_{\mathrm{rf}}=\mathbf{I}_{2}, \\
\mathbf{m}_{\mathrm{rf}}=\mathbf{n}_{\mathrm{rf}}=\mathbf{0}, \\
\mathbf{N}_{\mathrm{rf}}=\left(\begin{array}{cc}
1 & 0 \\
\tilde{V} & 1
\end{array}\right),
\end{gathered}
$$

where $\tilde{V}$ was defined in Eq. (4).

The synchrotron tune as a function of $\tilde{V}$ is,

$$
Q_{s}=\frac{1}{2 \pi} \sqrt{\frac{-L \eta_{\gamma} \tilde{V}}{4 \pi^{2} \beta_{r}^{2}} \cos \Psi_{0}},
$$

where $\eta_{\gamma}$ is the slip factor.

Evaluating Eqs. (44) and (45) for $\mathbf{T}_{10} \mathbf{T}_{\mathrm{RF}} \mathbf{T}_{01}$ yields,

$$
\begin{aligned}
\zeta_{a 0}= & \frac{\tilde{V}}{a \chi}\left[\eta_{x 1}\left(\cos \Delta \phi_{01}-\cos \left(\nu_{x}-\Delta \phi_{01}\right)\right)\right. \\
& \left.-\beta_{x} \eta_{x 1}^{\prime}\left(\sin \Delta \phi_{01}+\sin \left(\nu_{x}-\Delta \phi_{01}\right)\right)\right]
\end{aligned}
$$

$$
\begin{aligned}
\eta_{a 0}= & \frac{2}{a \chi}\left(1-\cos \nu_{x}\right) \eta_{x 0} \\
& -\frac{\tilde{V}}{a \chi}\left[\left(L_{01} \alpha_{01}^{p}+L_{10} \alpha_{10}^{p}\right) \eta_{x 0}\right. \\
& +\left(L_{10} \alpha_{10}^{p} \cos \Delta \phi_{01}+L_{01} \alpha_{01}^{p} \cos \Delta \phi_{10}\right) \eta_{x 1} \\
& \left.-\left(L_{10} \alpha_{10}^{p} \sin \Delta \phi_{01}-L_{01} \alpha_{01}^{p} \sin \Delta \phi_{10}\right) \beta_{x} \eta_{x 1}^{\prime}\right]
\end{aligned}
$$

where $\chi=\sqrt{\operatorname{Tr}(\mathbf{M}-\mathbf{N})^{2}+4\left|\mathbf{m}+\mathbf{n}^{\dagger}\right|}$.

We find that as anticipated $\zeta_{a 0}$ is proportional to the dispersion in the cavity and the accelerating voltage.

In the absence of $x z$ coupling, $\left|\mathbf{m}+\mathbf{n}^{\dagger}\right|=0$. In that case, $\chi=2 \cos \nu_{x}-2 \cos \nu_{s}$ and $a=1$. As rf voltage goes to zero, which implies a small $\nu_{s}$, the normal-mode dispersion $\eta_{a 0}$ becomes the ordinary dispersion $\eta_{x 0}$.

\section{LATTICE DESIGN}

In a storage ring with multiple sets of rf cavities, $\zeta_{a 0}$ and $\eta_{a 0}$ at the instrumentation source point are given by the sum of the contributions from each cavity.

For a given pair of cavities, assume that the coupling is small such that $\left|\mathbf{m}+\mathbf{n}^{\dagger}\right|$ is small. Then $\chi_{1} \approx \chi_{2}$, where subscripts 1 and 2 denote the separate cavities. Further assuming that $\eta_{1}=\eta_{2}$ and $\eta_{1}^{\prime}=\eta_{2}^{\prime}$, we find that

$$
\sum \zeta_{a 0} \propto \cos \frac{\Delta \phi_{12}}{2}
$$

where $\Delta \phi_{12}$ is the betatron phase advance between the cavities. A phase advance of $(2 n+1) \frac{\pi}{2}$ causes the $\zeta_{0}$ generated in one cavity to cancel out the $\zeta_{0}$ generated in the other cavity.

In the case of a ring with only one rf cavity, or if the $(2 n+1) \frac{\pi}{2}$ condition cannot be met, $\zeta_{a}$ can be minimized at the instrumentation source point by adjusting the betatron phase advance such that the tilt passes through a zero at the instrumentation source point.

In CESR, there are two pairs of rf cavities, separated by about 1.5 betatron wavelengths. Because $\eta_{1}, \eta_{1}^{\prime}$, and $\chi_{1}$ are only approximately equal to $\eta_{2}, \eta_{2}^{\prime}$, and $\chi_{2}, \zeta_{a}$ at the observation point is minimized using a optimizer that varies quadrupole strengths. The minimization procedure results in an approximately closed $x z$ coupling bump through the south region of the accelerator.

As shown in Eq. (38), the beam tilt has a contribution from $\alpha_{c}$, in addition to $\zeta_{a}$. As shown in Fig. $4, \alpha_{c}$ is naturally zero at the horizontal beam size measurement source point for CesrTA.

A second method to eliminate $\zeta_{a}$ is to zero the horizontal dispersion in the rf cavities. In CESR this forces nonzero dispersion in the nearby damping wiggler straight, and therefore requires that 6 of the 12 wigglers be powered off. In Fig. 1, these are the south end wiggler triplets at six o'clock. 


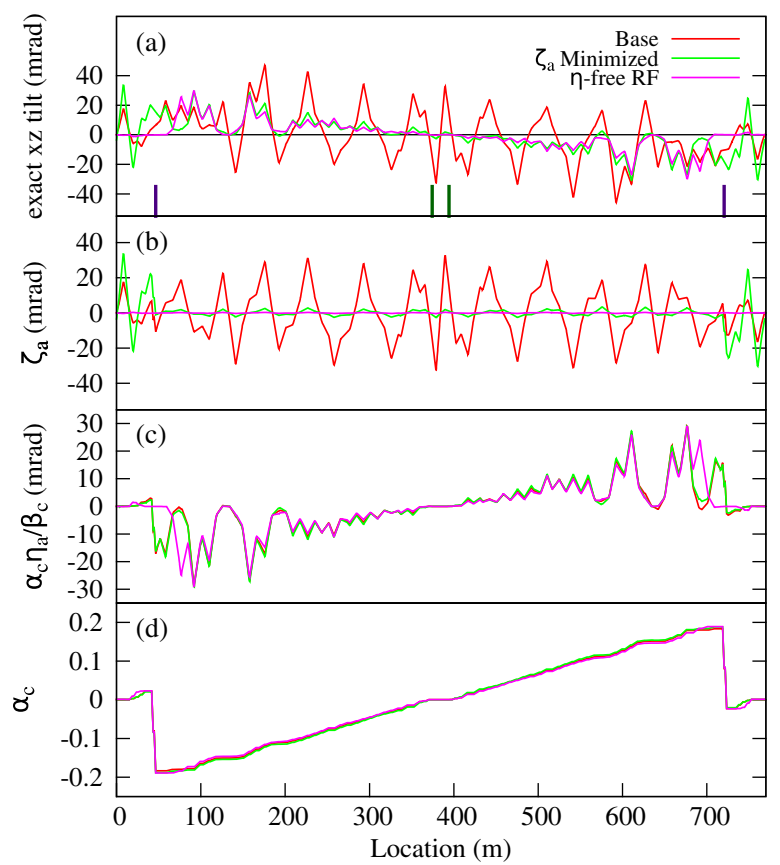

FIG. 4. For lattices with 6.3 MV total rf voltage: (a) Tilt of beam in $x z$ plane calculated from major axis of projected beam envelope. Purple bars indicate the location of the two pairs of RF cavities. Green bars indicate the location of horizontal beam size monitors. (b) Crabbing contribution from 1st term in Eq. (38). (c) Crabbing contribution from 2nd term in Eq. (38). (d) Longitudinal Twiss parameter $\alpha_{c}$.

Figure 4 shows the model $\zeta_{a}$ values for the base lattice, the lattice with $\zeta_{a}$ minimized, and a lattice with zero dispersion in the rf cavities. "Base" refers to the base CesrTA lattice. This lattice has $\sim 1 \mathrm{~m}$ horizontal dispersion in each pair of rf cavities. " $\zeta_{a}$ minimized" refers to the lattice optimized to minimize the effect of crabbing at the instrumentation source points. " $\eta$ free" refers to the lattice with zero dispersion in the rf cavities.

The zero current horizontal and vertical emittances of the " $\eta$ free" lattice are larger because 6 of the 12 damping wigglers are powered off. The damping time of the "Base", " $\zeta_{a}$ minimized", and " $\eta$ free" lattices are 56.6, 56.6, and $99.8 \mathrm{~ms}$, respectively.

\section{EXPERIMENT}

Measurements are taken with each of the three lattices at $2.1 \mathrm{GeV}$ using a single bunch of positrons. The experiment is conducted by setting the rf voltage, then taking several bunch size measurements at both 0.5 and $1.0 \mathrm{~mA}$. Horizontal and vertical beam size and bunch length are recorded from 6.3 MV down to $1.0 \mathrm{MV}$ in roughly $1 \mathrm{MV}$ increments. The total $\mathrm{rf}$ voltage is split roughly equally among the four rf cavities.

Vertical beam size is measured by imaging $\mathrm{x}$-rays from a hard bend onto a vertical diode detector array [10,11]. Horizontal beam size is measured using an interferometer
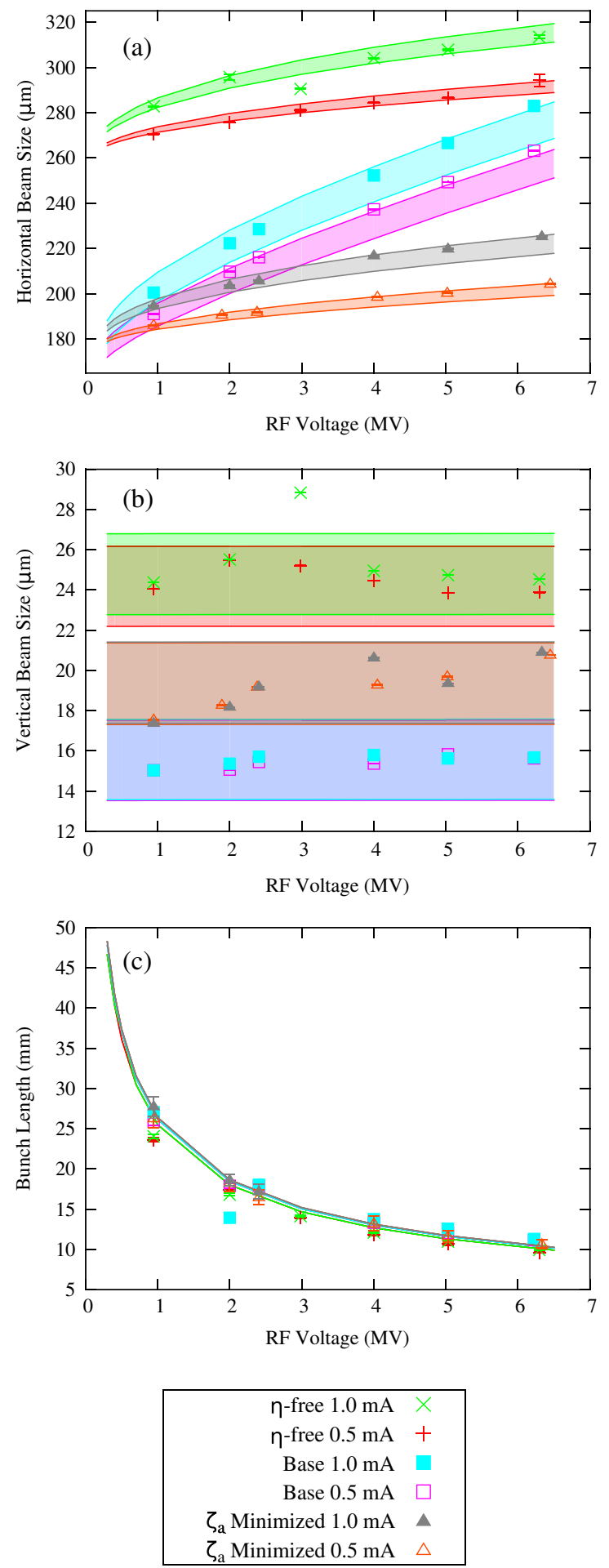

FIG. 5. (a) Horizontal, (b) vertical, and (c) bunch length measurements and simulation results. Points are data and curves are simulation results. The data points are the average over several measurements and the error bars are the statistical uncertainty. In CesrTA, $1.0 \mathrm{~mA}=1.6 \times 10^{10}$ particles/bunch.

which images synchrotron radiation from a soft bend [12]. Bunch length measurements are made with a streak camera using synchrotron radiation from the same bend [13]. 
The simulation includes IBS calculated using the KuboOide formalism [14]. IBS occurs when collisions among the particles that compose a beam transfer momentum between the particles such that the emittance of the beam is changed. The implementation of this formalism at CesrTA is discussed in [15].

Figure 5 shows the measured horizontal, vertical, and longitudinal bunch size, along with simulation results. Beam sizes are obtained from the simulation by projecting the beam envelope into lab frame coordinates using Eq. (31).

The simulation takes as parameters the zero current $a$-mode and $b$-mode emittances. These emittances are obtained by fitting the data.

The vertical beam size measurement is subject to a $\pm 2 \mu \mathrm{m}$ systematic uncertainty. This is accounted for by running the simulation twice, adjusting the $b$-mode emittance parameter to span a $4 \mu \mathrm{m}$ range of vertical beam size. This parameter range is the source of the colored bands in the plots.

The horizontal beam size in the "Base" lattice has an additional source of systematic uncertainty due to the beam tilt. The dependence of the projected horizontal beam size $\sigma_{x}$ on the $x z$ tilt angle $\theta_{x z}$ is

$$
\frac{d \sigma_{x}}{d \theta_{x z}}=\frac{\theta_{x z} \sigma_{z}^{2}}{\sigma_{x}}
$$

where $\sigma_{z}$ is the bunch length. At the horizontal beam size monitor source point,

$$
\begin{gathered}
\frac{d \sigma_{x}}{d \theta_{x z}}=6.6 \frac{\mu \mathrm{m}}{\mathrm{mrad}}, \\
\frac{d \theta_{x z}}{d s}=-1.4 \frac{\mathrm{mrad}}{\mathrm{m}} .
\end{gathered}
$$

The uncertainty in the location of the source point, given by the depth of field, is $0.35 \mathrm{~m}$ [12]. This contributes an additional $\pm 3.2 \mu \mathrm{m}$ systematic uncertainty to the "Base" lattice simulation results.

For the " $\zeta_{a}$ minimized" lattice, the same zero current $a$-mode emittance and $b$-mode emittance is used for all data points. This is true also for the " $\eta$-free" lattice.

The presence of $\zeta_{a}$ in a storage ring affects the zero current $a$-mode emittance. The dependence of the zero current emittance on rf voltage is estimated using PTC [16]. This dependence is significant only for the "Base" lattice.

Table II shows the emittances used to generate the simulation results.

The differences in the measurement results between 0.5 and $1.0 \mathrm{~mA}$ are due to IBS. The effect is most noticeable in the horizontal (Fig. 5a) due to the large amount of horizontal dispersion throughout the ring. The rms horizontal dispersion is $1 \mathrm{~m}$.
TABLE II. Simulation parameters: zero current emittances. The presence of $\zeta_{a}$ in the "Base" lattice creates a rf voltage dependent zero current $a$-mode emittance.

\begin{tabular}{lccc}
\hline \hline Lattice & $V_{\mathrm{rf}}$ & $\epsilon_{a}(\mathrm{~nm})$ & $\epsilon_{b}$ \\
\hline$\eta$-free & All & 5.2 & $12.3 \mathrm{pm}-17.9 \mathrm{pm}$ \\
$\zeta_{a}$ minimized & All & 3.3 & $7.3 \mathrm{pm}-11.1 \mathrm{pm}$ \\
& $<1.0 \mathrm{MV}$ & 2.99 & \\
& $1.0 \mathrm{MV}$ & 3.00 & \\
Base & $2.0 \mathrm{MV}$ & 3.02 & \\
& $3.0 \mathrm{MV}$ & 3.05 & $4.58 \mathrm{pm}-7.69 \mathrm{pm}$ \\
& $4.0 \mathrm{MV}$ & 3.08 & \\
& $5.0 \mathrm{MV}$ & 3.13 & \\
& $6.5 \mathrm{MV}$ & 3.21 & \\
\hline \hline
\end{tabular}

The jump in vertical beam size at 3 MV for the " $\eta$ free" lattice at $1.0 \mathrm{~mA}$ (Fig. 5b) was due to crossing a synchrobetatron resonance.

The model lattices used for the simulation are ideal, with no vertical dispersion or transverse coupling. The result is that the simulation predicts negligible IBS blow up in the vertical dimension. The insensitivity of the measured vertical beam size to changes in current and bunch length for the "Base" and " $\eta$ free" lattices suggest that transverse coupling and vertical dispersion are well corrected. The vertical dispersion is measured to be less than $15 \mathrm{~mm}$. The coupling is measured using an extended Edwards-Teng formalism to be $\bar{V}_{12}<0.003$.

An upward trend is suggested in the vertical beam size for the " $\zeta_{a}$ minimized" lattice (Fig. 5b). It is unlikely that this trend is due to IBS, as the beam size is the same for the 0.5 and $1.0 \mathrm{~mA}$ data points. Such a trend could arise from an optics error introducing $y z$ coupling.

\section{CONCLUSION}

The decomposition of the 1-turn matrix into the coupling matrices $\mathbf{H}$ and $\mathbf{V}$ and Twiss matrix $\mathbf{B}$ yields useful information about the coupling properties of a ring. We showed Eq. (38) that the beam has an $x z$ tilt given by the crabbing dispersion $\zeta_{a}$ and a term that is proportional to the local horizontal dispersion. We showed how the presence of dispersion in the rf cavities can generate crabbing dispersion throughout the ring.

The beam size measurements vs rf voltage from the " $\eta$ free" and " $\zeta_{a}$ minimized" lattices agree well with simulation. The dependence of the measured horizontal beam size on rf voltage is due entirely to IBS effects. The residual tilt after correction is negligible. Evidently, our methods for mitigating $x z$ tilt are effective.

For the "Base" lattice, which has significant $\zeta_{a}$ throughout, $x z$ tilt makes a nonnegligible contribution to projected horizontal beam size. Agreement between the "Base" lattice beam size measurements and simulation result is reasonable and suggests that the projected size of coupled beams can be reliably computed using Eq. (31). 
As described in Sec. I, crabbing affects beam size measurements and has potential applications in mitigating the effects of angle crossing in a collider, and in generating subpicosecond pulses in a light source. In Sec. II and Sec. III we explained through two different methods how dispersion in ordinary rf cavities generates crabbing and how the tilt angle of the beam can be calculated. In Sec. IV we presented methods for controlling, or mitigating, the amount of tilt. This was followed by Sec. V, where we supported our theory with experiment.

\section{ACKNOWLEDGMENTS}

The experiments reported here would not have been possible without the diligent support of the CESR Operations Group. This research was supported by NSF and DOE Contracts No. PHY-0734867, No. PHY-1002467, No. PHYS-1068662, No. DE-FC02-08ER41538, and No. DE-SC0006505.

[1] T. Abe, K. Akai, M. Akemoto et al., in Proceedings of the 22nd Particle Accelerator Conference, PAC-2007, Albuquerque, NM (IEEE, New York, 2007), p. 27.

[2] M. Palmer et al., in Proceedings of the 23rd Particle Accelerator Conference, Vancouver, Canada, 2009 (IEEE, Piscataway, NJ, 2009), p. 4200.

[3] D. Sagan, Nucl. Instrum. Methods Phys. Res., Sect. A 558, 356 (2006).

[4] R. Calaga, R. Toms, and F. Zimmermann, in Proceedings of the International Particle Accelerator Conference, Kyoto, Japan (ICR, Kyoto, 2010), p. 1240.
[5] A. Zholents, P. Heimann, M. Zolotorev, and J. Byrd, Nucl. Instrum. Methods Phys. Res., Sect. A 425, 385 (1999).

[6] D. A. Edwards and L. C. Teng, IEEE Trans. Nucl. Sci. 20, 885 (1973).

[7] D. Sagan and D. Rubin, Phys. Rev. ST Accel. Beams 2, 074001 (1999).

[8] K. Ohmi, K. Hirata, and K. Oide, Phys. Rev. E 49, 751 (1994).

[9] A. Wolski, Phys. Rev. ST Accel. Beams 9, 024001 (2006).

[10] N. T. Rider, M. G. Billing, M. P. Ehrlichman, D. P. Peterson, D. Rubin, J. P. Shanks, K. G. Sonnad, M. A. Palmer, and J. W. Flanagan, in Proceedings of the International Beam Instrumentation Conference 2012, Tsukuba, p. 585.

[11] J.P. Alexander and D. P. Peterson, in The Handbook of Accelerator Physics and Engineering 2nd Edition, edited by A. W. Chao, K. H. Mess, M. Tigner, and F. Zimmerman (World Scientific, Singapore, 2013), p. 721.

[12] S. T. Wang, D. Rubin, J. Conway, M. Palmer, D. Hartill, R. Campbell, and R. Holtzapple, Nucl. Instrum. Methods Phys. Res., Sect. A 703, 80 (2013).

[13] R. Holtzapple, M. Billing, D. Hartill, M. Stedinger, and B. Podobedov, Phys. Rev. ST Accel. Beams 3, 034401 (2000).

[14] K. Kubo and K. Oide, Phys. Rev. ST Accel. Beams 4, 124401 (2001).

[15] M. P. Ehrlichman, W. Hartung, B. Heltsley, D. P. Peterson, N. Rider, D. Rubin, D. Sagan, J. Shanks, S. T. Wang, R. Campbell, and R. Holtzapple, Phys. Rev. ST Accel. Beams 16, 104401 (2013).

[16] E. Forest, Beam Dynamics: A New Attitude and Framework (Harwood Academic Publishers, Chur, Switzerland, 1998). 